\title{
ON BOUNDED ANALYTIC FUNCTIONS
}

\section{ZEEV NEHARI}

The objective of this paper is to give an alternative derivation of results on bounded analytic functions recently obtained by Ahlfors [1] and Garabedian [2]. ${ }^{1}$ While it is admitted that the main idea to be used is more in the nature of a lucky guess than of a method, it will be found that the gain in brevity and simplicity of the argument is considerable. As a by-product, we shall also obtain a number of hitherto unknown identities between various domain functions.

The basic problem treated in the above-mentioned papers is the following generalization of the classical Schwarz lemma: Given a finite schlicht domain $D$ of connectivity $n(n \geqq 1)$ in the complex $z$ plane and a point $\zeta$ in $D$, to find a function $F(z)$ with the following properties: (a) $F(z)$ belongs to the family $B$ of analytic functions $f(z)$ which are single-valued and regular in $D$ and satisfy there $|f(z)| \leqq 1$; (b) $\left|F^{\prime}(\zeta)\right| \geqq\left|f^{\prime}(\zeta)\right|$, where $f(z)$ is any function in $B$. Evidently, it is sufficient to solve this problem for any domain $D^{\prime}$ which is conformally equivalent to $D$. In particular, we may therefore assume, without restricting the generality of what follows, that $D$ is bounded by analytic curves.

It was shown by Ahlfors that $F(z)$ yields a $(1, n)$ conformal mapping of $D$ onto the interior of the unit circle and that $n-1$ of the $n$ zeros of $F(z)$ coincide with the zeros of a single-valued function $h(z)$ which is regular in $D$ with the exception of a simple pole at $z=\zeta$ and satisfies $-i h(z) d z>0$ on the boundary $\Gamma$ of $D$; the $n$th zero of $F(z)$ is located at $z=\zeta$. It was subsequently noticed by Garabedian that the function $h(z)$ can be written in the form $h(z)=F(z) q(z)$ where $q(z)-(z-\zeta)^{-2}$ is regular in $D$ and that the extremal property of $F(z)$ can be deduced in a very elegant manner from the resulting inequality

$$
\frac{1}{i} F(z) q(z) d z>0, \quad z \in \Gamma .
$$

Indeed, if $f(z)$ is in $B$ and is continuous on $\Gamma$, we have, by the residue theorem,

$$
\left|f^{\prime}(\zeta)\right|=\left|\frac{1}{2 \pi i} \int_{\mathbf{r}} f(z) q(z) d z\right| \leqq \frac{1}{2 \pi} \int_{\mathbf{\Gamma}}|q(z) d z|=\frac{1}{2 \pi} \int_{\mathbf{\Gamma}}|F(z) q(z) d z|
$$

Received by the editors October 19, 1948 and, in revised form, January 8, 1949.

1 Numbers in brackets refer to the references cited at the end of the paper. 


$$
=\frac{1}{2 \pi i} \int_{\Gamma} F(z) q(z) d z=F^{\prime}(\zeta),
$$

where (1) and the fact that, on $\Gamma,|F(z)| \equiv 1$ have been used.

The inequality (1) not only solves the problem stated above but, as likewise shown by Garabedian, it may also be used in order to establish the connection between $F(z)$ and the Szegö kernel function [4] of $D$. This connection, in turn, leads to a possibility of effectively computing $F^{\prime}(\zeta)$ in terms of a certain complete orthonormal system of functions in $D$.

Everything depends therefore on the proof of the existence of two functions $F(z)$ and $q(z)$, where $q(z)$ has the required double pole and $|F(z)| \equiv 1$ on $\Gamma$, such that (1) is satisfied for $z \in \Gamma$. We shall carry through this proof by giving, with the help of an allied simple extremal problem, a construction of these functions in terms of the Green's function and the harmonic measures of $D$.

We use the following notations: $p(z)=p(z, \zeta)=g(z, \zeta)+i h(z, \zeta)$ denotes the analytic function whose real part is the Green's function $g(z, \zeta)$ of $D$, that is, the harmonic function which is regular in $D$, apart from the point $z=\zeta$ where $g(z, \zeta)+\log |z-\zeta|$ is regular, and vanishes for $z \in D$. $w_{\nu}(z)=\omega_{\nu}(z)+i \omega_{\nu}^{*}(z)$ denotes the analytic function whose real part is the harmonic measure $\omega_{\nu}(z)$ of the boundary component $\Gamma,(\nu=1,2, \cdots, n)$, that is, that harmonic function which is regular in $D$, is equal to 1 for $z \in \Gamma$, and vanishes on all the other boundary continua. We further use the notations $u(z)=u(z, \zeta)$ $=\partial p(z, \zeta) / \partial \xi, v(z)=v(z, \zeta)=(1 / i) \partial p(z, \zeta) / \partial \eta, \zeta=\xi+i \eta$. It is easily confirmed that, with the exception of a simple pole of residue 1 at $z=\zeta$, both $u(z)$ and $v(z)$ are free of singularities in $D$ and that, furthermore, $\operatorname{Re}\{u(z, \zeta)\} \equiv \operatorname{Im}\{v(z, \zeta)\} \equiv 0$ for $z \in \Gamma$.

Since the real parts of the functions $p(z), w_{p}(z), u(z), i v(z)$ are constant on $\Gamma$, the differentials

$$
\frac{1}{i} p^{\prime}(z) d z, \quad \frac{1}{i} w_{\nu}^{\prime}(z) d z, \quad \frac{1}{i} u^{\prime}(z) d z, \quad v^{\prime}(z) d z
$$

are real there. The same is therefore also true of a linear combination

$$
\begin{aligned}
& \frac{1}{i} t(z) d z \\
& \quad=\frac{1}{i}\left[\cos 2 \lambda u^{\prime}(z)+i \sin 2 \lambda v^{\prime}(z)-\alpha p^{\prime}(z)+\sum_{p=1}^{n-1} \mu_{\nu} w_{\nu}^{\prime}(z)\right] d z
\end{aligned}
$$

with real $\lambda, \alpha, \mu_{1}, \cdots, \mu_{n-1}$. Since $\Gamma$ consists of analytic curves, the 
functions $u(z), v(z), p(z), w_{\nu}(z)$ are regular on $\Gamma$-an immediate consequence of the Schwarz reflection principle. It is further well known, and easily proved, that $i p^{\prime}(z) d z$ is not only real but also positive on $\Gamma$. If, in (2), $\lambda, \mu_{1}, \cdots, \mu_{n-1}$ are kept constant and $\alpha(\alpha>0)$ is taken large enough, the differential $t(z) d z$ will therefore satisfy

$$
\frac{1}{i} t(z) d z \geqq 0, \quad z \in \Gamma .
$$

We now pose the following extremal problem: For given $\lambda$, to find the differential (2) which minimizes $\alpha$ under the condition (3). It was shown by the author [3] that this problem has a solution, say $i^{-1} t_{\lambda}(z) d z$, and that the function $t_{\lambda}(z)$ belonging to this extremal differential has a double zero on each boundary component $\Gamma_{\nu}, \nu$ $=1,2, \cdots, n$.

It is readily confirmed that, in the vicinity of $z=\zeta$, the function $t_{\lambda}(z)$ has the expansion

$$
t_{\lambda}(z)=-\frac{e^{2 i \lambda}}{(z-\zeta)^{2}}-\frac{\alpha}{z-\zeta}+\text { regular terms. }
$$

We shall now show that $t_{\lambda}(z)$ is free of zeros in $D$. In view of (3), the expression $t_{\lambda}(z) d z$ does not change its argument if $z$ describes the boundary $\Gamma$ of $D$. If $\Delta \arg t_{\lambda}$ denotes the total change of the argument of $t_{\lambda}$ along $\Gamma$, and $\Delta$ arg $d z$ has a similar meaning, we have therefore

$$
\Delta \arg t_{\lambda}=-\Delta \arg d z .
$$

Since $\Delta \arg d z=-2 \pi(n-2)$, it follows that

$$
\frac{1}{2 \pi} \Delta \arg t_{\lambda}=n-2 \text {. }
$$

By the argument principle, this is equal to the number of zeros of $t_{\lambda}$ in $D$ minus the number of its poles there, plus a contribution of $1 / 2$ for each zero of $t_{\lambda}$ on $\Gamma$. Since $t_{\lambda}$ has $n$ double zeros on $\Gamma$ and a double pole at $z=\zeta$, it follows from (5) that $t_{\lambda}$ has no zeros in $D$ and, moreover, that $t_{\lambda}$ does not vanish on $\Gamma$ at points different from the $n$ double zeros already located.

Our next step is to prove that the function

$$
\sigma_{\lambda}(z)=\left(t_{\lambda}(z)\right)^{1 / 2}=\frac{i e^{\lambda}}{z-\zeta}+\text { regular terms }
$$

is single-valued in $D$. For this purpose we have to show that, in addition to the fact just established that $\sigma_{\lambda}(z)$ has no zeros in $D$, the argu- 
ment of the function $\sigma_{\lambda}(z)$ returns to its initial value if $z$ describes a boundary component $\Gamma_{\nu}(\nu=1, \cdots, n)$ of $D$. This is equivalent to showing that the increment of $\arg t_{\lambda}$ along $\Gamma_{\nu}$ is an even multiple of $2 \pi$. That this is indeed the case is easily shown as follows: If we start from the point on $\Gamma$, at which the double zero of $t_{\lambda}$ is located and describe $\Gamma$, in the positive sense with respect to $D$, the total variation of $\arg t_{\lambda}$ is, in view of (3), equal to the negative value of the total variation of $\arg d z$, that is, it is either $2 \pi$ or $-2 \pi$. At the double zero of $t_{\lambda}$, arg $t_{\lambda}$ jumps by $2 \pi$. The variation of $t_{\lambda}$ along the whole of $\Gamma_{\nu}$ is therefore either 0 or $4 \pi$. Hence, the function $\sigma_{\lambda}(z)$ defined in (6) is indeed regular and single-valued in $D$.

We now introduce two functions $K_{\lambda}(z, \zeta)$ and $L_{\lambda}(z, \zeta)$ by the definitions

$$
\begin{aligned}
& K(z, \zeta)=K_{\lambda}(z, \zeta)=\frac{i e^{i \lambda}}{4 \pi}\left(\sigma_{\lambda}+i \sigma_{\lambda+\pi / 2}\right) \\
& L(z, \zeta)=L_{\lambda}(z, \zeta)=\frac{e^{-i \lambda}}{4 \pi i}\left(\sigma_{\lambda}-i \sigma_{\lambda+\pi / 2}\right)
\end{aligned}
$$

Both functions have also been written without the subscript $\lambda$, in anticipation of the fact-to be proved further below-that they are independent of the parameter $\lambda$. It is easily confirmed that $K(z, \zeta)$ is regular in $D$ and that $L(z, \zeta)$ has a simple pole at $z=\zeta$ with the residue $(2 \pi)^{-1}$.

For $z \in \Gamma$, the two functions $K(z)=K(z, \zeta)$ and $L(z)=L(z, \zeta)$ are connected by the relation

$$
\frac{1}{i} L(z) d z=K^{*}(z) d s,
$$

where $d s=|d z|$ is the length element on $\Gamma .^{2}$ We shall prove (8) by showing that both the arguments and the absolute values on both sides are equal. We have, by (6), (7) and (3),

$$
\frac{1}{i} K(z) L(z) d z=\frac{1}{16 \pi^{2}}\left(\sigma_{\lambda}^{2}+\sigma_{\lambda+\pi / 2}^{2}\right) d z \geqq 0,
$$

whence

$$
\arg \frac{1}{i} L(z) d z=-\arg K(z)=\arg K^{*}(z)=\arg K^{*}(z) d s,
$$

which shows that the arguments are equal. Again by (7), we have

2 The asterisk denotes conjugate complex quantities. 


$$
\frac{K(z)}{L(z)}=-e^{2 \lambda}\left(\frac{\sigma_{\lambda}}{\sigma_{\lambda+\pi / 2}}+i\right)\left(\frac{\sigma_{\lambda}}{\sigma_{\lambda+\pi / 2}}-i\right)^{-1} .
$$

In view of (3) and (6), we have

$$
\left(\frac{\sigma_{\lambda}}{\sigma_{\lambda+\pi / 2}}\right)^{2} \doteq \frac{(1 / i) t_{\lambda} d z}{(1 / i) t_{\lambda+\pi / 2} d z} \geqq 0
$$

As a result, $\sigma_{\lambda} \sigma_{\lambda+\pi / 2}^{-1}$ is real on $\Gamma$, whence, by (10), $\left|K L^{-1}\right| \equiv 1$ for $z \in \Gamma$. This completes the proof of (8).

As a first application of (8), we shall show that $K(z)$ and $L(z)$ are independent of $\lambda$ and that we were therefore justified in dropping the subscript $\lambda$ in (7). For suppose there are two different pairs of functions $K_{1}, L_{1}$ and $K_{2}, L_{2}$ belonging to different values of $\lambda$. Obviously, the function $L_{1}-L_{2}$ is regular in $D+\Gamma$, whence, by Cauchy's theorem,

$$
\int_{\Gamma}\left[L_{1}(z)-L_{2}(z)\right]\left[K_{1}(z)-K_{2}(z)\right] d z=0 .
$$

In view of (8), this is equivalent to

$$
\int_{\Gamma}\left|K_{1}(z)-K_{2}(z)\right|^{2} d s=0,
$$

whence $K_{1}(z) \equiv K_{2}(z)$ on $\Gamma$ and therefore throughout $D$. The identity $L_{1}(z) \equiv L_{2}(z)$ follows by another application of (8).

The fact that both $K(z)$ and $L(z)$ are independent of $\lambda$ leads to an interesting identity. With the notations

$$
\sigma(z)=\sigma_{0}(z), \quad \tau(z)=\sigma_{\pi / 2}(z)
$$

we have, by (7),

$$
\begin{aligned}
e^{i \lambda}\left(\sigma_{\lambda}+i \sigma_{\lambda+\pi / 2}\right) & =\sigma+i \tau \\
e^{-i \lambda}\left(\sigma_{\lambda}-i \sigma_{\lambda+\pi / 2}\right) & =\sigma-i \tau
\end{aligned}
$$

whence

$$
\sigma_{\lambda}(z)=\cos \lambda \sigma(z)+\sin \lambda \tau(z) .
$$

Consider now the function

$$
T(z)=\sigma(z) / \tau(z) .
$$

By (11) and (12), $T(z)$ is real on $\Gamma$. At interior points of $D, T(z)$ cannot be real. Indeed, suppose $T(z)=-\tan \lambda_{0}$, where $\lambda_{0}$ is an arbitrary real number between $-\pi / 2$ and $\pi / 2$. This would entail $\cos \lambda_{0} \sigma(z)$ 
$+\sin \lambda_{0} \tau(z)=0$. By (13), this would mean that $\sigma_{\lambda_{0}}(z)$ has a zero in $D$, which further above was shown not to be true. The same argument also shows that every real value is taken by $T(z)$ on $\Gamma$ exactly $n$ times, this being the number of zeros of $\sigma_{\lambda}(z)$ on $\Gamma$. Since $T(z)$ is real on $\Gamma$ but at no interior point of $D$, it follows therefore that $w=T(z)$ maps $D$ onto an $n$ times covered half-plane which is bounded by the real axis. If, in (6), the positive value of the square root is taken, we have, by (6) and (12), $T(\zeta)=-i$, which shows that $w=T(z)$ yields a $(1, n)$ mapping of $D$ onto the lower half-plane, $\operatorname{Im}\{w\}<0$. Consequently, the function

$$
F(z)=-\frac{T(z)+i}{T(z)-i}=-\frac{\sigma(z)+i \tau(z)}{\sigma(z)-i \tau(z)}=\frac{K(z)}{L(z)}
$$

yields a $(1, n)$ mapping of $D$ onto the unit circle. This, together with (9) and the argument principle, incidentally shows that $K(z)$ has $n-1$ zeros in $D$ and that $L(z)$ is free of zeros there.

If we introduce the function $q(z)$ by

$$
q(z)=4 \pi^{2} L^{2}(z)
$$

we have, by (14) and (9),

$$
\frac{1}{i} F(z) q(z) d z=\frac{4 \pi^{2}}{i} K(z) L(z) d z \geqq 0, \quad z \in \Gamma .
$$

Comparison of (16) with (1) shows that $F(z)$ and $q(z)$ are identical with the functions $F(z)$ and $q(z)$ introduced there, provided we can show that the principal part of $q(z)$ at $z=\zeta$ is $(z-\zeta)^{-2}$. This is indeed the case. For suppose the principal part of $q(z)$ at this point is $(z-\zeta)^{-2}+\gamma(z-\zeta)^{-1}$. By the residue theorem and in view of $|F(z)|$ $\equiv 1(z \in \Gamma)$, we have

$$
\gamma=\frac{1}{2 \pi i} \int_{\mathbf{r}} q(z) d z=\frac{1}{2 \pi i} \int_{\mathbf{r}} F^{*}(z) F(z) q(z) d z .
$$

By virtue of (16), this may also be written

$$
\gamma^{*}=\frac{1}{2 \pi i} \int_{\mathbf{r}} F^{2}(z) q(z) d z .
$$

Since $F(z)$ has a zero at $z=\zeta$-originating from the simple pole of $L(z)$ - the integral at the right-hand side vanishes by the Cauchy theorem, whence $\gamma=0$. This completes the proof of the identity of the functions $F(z)$ and $q(z)$ with the functions-denoted by the same symbols-introduced at the beginning of this paper. 
It was shown by Garabedian that the function $(2 \pi)^{-1} F(z)[q(z)]^{1 / 2}$ is identical with the Szegö kernel function [4] of $D$. In view of (14) and (15), this function is identical with $K(z)$. The identity of $K(z)$ $=K(z, \zeta)$ with the Szegö kernel function is also easily shown with the help of (8). Indeed, if $f(z)$ is a function which is regular and singlevalued in $D$ and continuous in $D+\Gamma$, we have, by (8) and the Cauchy theorem,

$$
f(\zeta)=\frac{1}{i} \int_{\Gamma} f(z) L(z) d z=\int_{\Gamma} K^{*}(z, \zeta) f(z) d s,
$$

and this relation is characteristic cf the Szegö kernel function.

The Szegö kernel function $K(z, \zeta)$ can be effectively computed in terms of a certain complete orthonormal set of functions [4]. If $K(z, \zeta)$ is known, $L(z)=L(z, \zeta)$ can also be immediately determined. We have, by (8) and the residue theorem,

$$
\int_{\Gamma} \frac{K^{*}(t, \zeta) d s_{t}}{t-z}=\frac{1}{i} \int_{\Gamma} \frac{L(t, \zeta) d t}{t-z}=\frac{1}{\zeta-z}+2 \pi L(z, \zeta),
$$

whence

$$
2 \pi L(z, \zeta)=\frac{1}{z-\zeta}+\int_{\Gamma} \frac{K^{*}(t, \zeta) d s_{t}}{t-z}
$$

Once the kernel function is known, we are thus in a position to determine explicitly the functions $F(z)$ and $q(z)$, as well as the functions $\sigma_{\lambda}(z)$ mentioned before.

If we combine (2), (6), (7), (13), (14), (15), (18), a large number of interesting identities connecting $K(z), L(z), F(z), q(z), \sigma_{\lambda}(z)$ with the Green's function and the harmonic measures can be obtained. As an example, we prove the identity

$$
\frac{2 \pi K(z, \zeta) L(z, \zeta)}{K(\zeta, \zeta)}=p^{\prime}(z, \zeta)+\sum_{v=1}^{n-1} c_{\nu} w_{\nu}^{\prime}(z)
$$

where, as in (2), $\operatorname{Re}\{p(z, \zeta)\}$ is the Green's function of $D, \operatorname{Re}\left\{w_{p}(z)\right\}$ the harmonic measures of $\Gamma_{\nu}(\nu=1, \cdots, n-1)$ with respect to $D$, and the $c_{\nu}$ are suitable real constants. Indeed, by (9), (6), (2), and (12)

$$
\frac{2 \pi K(z, \zeta) L(z, \zeta)}{K(\zeta, \zeta)}=\frac{\sigma^{2}(z)+\tau^{2}(z)}{8 \pi K(\zeta, \zeta)}=C p^{\prime}(z, \zeta)+\sum_{\nu=1}^{n-1} c_{\nu} w_{\nu}^{\prime}(z),
$$

where $C, c_{1}, c_{2}, \cdots, c_{n-1}$ are constants. Since the residue of the simple 
pole of $L(z, \zeta)$ at $z=\zeta$ is $(2 \pi)^{-1}$, the value of constant $C$ is immediately seen to be 1 . This completes the proof of the identity (19).

The constants $c$, in (19) can be expressed in terms of the harmonic measures $\omega_{\nu}(z)$, their periods $P_{\mu \nu}$ with respect to the boundary components $\Gamma_{\mu}$, and the kernel function. By (8), we have

$$
\int_{\Gamma_{\mu}} K(z, \zeta) L(z, \zeta) d z=\int_{\Gamma_{\mu}}|K(z, \zeta)|^{2} d s .
$$

On the other hand,

$$
\int_{\Gamma_{\mu}} p^{\prime}(z, \zeta) d z=2 \pi i \omega_{\nu}(\zeta)
$$

and

$$
\int_{\Gamma_{\mu}} w_{\nu}^{\prime}(z) d z=2 \pi i P_{\mu \nu}
$$

In view of (19), the constants $c_{\nu}$ are therefore the solutions of the system of linear equations

$$
[K(\zeta, \zeta)]^{-1} \int_{\Gamma_{\mu}}|K(z, \zeta)|^{2} d s=\omega_{\mu}(\zeta)+\sum_{\nu=1}^{n-1} P_{\mu \nu} c_{\nu}, \quad \mu=1, \cdots, n-1 .
$$

\section{REFERENCES}

1. L. V. Ahlfors, Bounded analytic functions, Duke Math. J. vol. 14 (1947) pp. $1-11$.

2. P. Garabedian, Schwarz' lemma and the Szegr kernel function, Thesis, Harvard University, 1948; Trans. Amer. Math. Soc. vol. 67 (1949) pp. 1-35.

3. Z. Nehari, Analytic functions possessing a positive real part, Duke Math. J. vol. 15 (1948) pp. 1033-1042.

4. G. Szegö, Über orthogonale Polynome, die zu einer gegebenen Kurve der komplexen Ebene gehören, Math. Zeit. vol. 9 (1921) pp. 218-270.

WASHINGTON UNIVERSITY 